\title{
Mathematical Modeling of Programmatic Requirements for Yaws Eradication
}

\author{
Michael Marks, Oriol Mitjà, Christopher Fitzpatrick, Kingsley Asiedu, \\ Anthony W. Solomon, David C.W. Mabey, Sebastian Funk
}

Yaws is targeted for eradication by 2020. The mainstay of the eradication strategy is mass treatment followed by case finding. Modeling has been used to inform programmatic requirements for other neglected tropical diseases and could provide insights into yaws eradication. We developed a model of yaws transmission varying the coverage and number of rounds of treatment. The estimated number of cases arising from an index case (basic reproduction number $\left[R_{0}\right]$ ) ranged from 1.08 to 3.32 . To have $80 \%$ probability of achieving eradication, 8 rounds of treatment with $80 \%$ coverage were required at low estimates of $R_{0}(1.45)$. This requirement increased to $95 \%$ at high estimates of $R_{0}(2.47)$. Extending the treatment interval to 12 months increased requirements at all estimates of $R_{0}$. At high estimates of $R_{0}$ with 12 monthly rounds of treatment, no combination of variables achieved eradication. Models should be used to guide the scale-up of yaws eradication.

Y aws is a bacterial infection caused by Treponema pallidum subsp. pertenue (1). The disease predominantly affects children living in poor, remote communities and results in lesions of the skin, bone, and cartilage. Previously, yaws was widespread throughout the tropics (2), but in the 20th century a series of control efforts based on mass treatment and case finding led by the World Health Organization (WHO) is estimated to have reduced the burden of cases worldwide by up to $95 \%$ (3). Despite these efforts, the disease has resurged in several countries in West and Central Africa, the Pacific, and Southeast Asia.

In 2012, a single dose of azithromycin was shown to be effective treatment for yaws (4). The availability of a well-tolerated oral agent has prompted WHO to develop a new eradication strategy, known as the Morges strategy, based on community mass azithromycin treatment (5). The

Author affiliations: Hospital for Tropical Diseases, London, UK (M. Marks, A.W. Solomon, D.C.W. Mabey); London School of Hygiene and Tropical Medicine, London (M. Marks, A.W. Solomon, D.C.W. Mabey, S. Funk); ISGlobal, Barcelona, Spain (O. Mitjà); World Health Organization, Geneva, Switzerland (C. Fitzpatrick, K. Asiedu, A.W. Solomon)

DOI: http://dx.doi.org/10.3201/eid2301.160487 strategy is supported by World Health Assembly resolution 66.12, which calls for eradication of yaws by 2020 (6). The strategy combines an initial round of total community treatment (TCT) followed by subsequent active case finding and total targeted treatment (TTT) of newly identified patients and their contacts. Pilot studies have shown that community mass treatment with azithromycin is a highly effective strategy for reducing the community prevalence of yaws $(7,8)$.

Data are limited to inform the optimum coverage and number of TCT or TTT rounds that are required to achieve elimination (i.e., interruption of transmission) of yaws at a local level to facilitate country-level elimination and ultimately global eradication. In India, a national yaws elimination campaign conducted during 1996-2004 resulted in substantial reduction in the prevalence of yaws, sustained interruption of transmission, and nationwide elimination (9). This program consisted of case-finding surveys and treatment with parenteral penicillin conducted every 6 months. Although this approach did not include the initial mass treatment round now recommended as part of the Morges strategy, its success indicates that serial rounds of high-coverage treatment might achieve local elimination.

A recent review of important research questions facing the global yaws eradication program has highlighted the need for more accurate data to inform the optimum number and coverage of rounds of TCT and TTT that will be required to achieve yaws eradication (10). Mathematical modeling has been used to inform control efforts for several other neglected tropical diseases (11-13) that are also managed by using community mass treatment strategies, and such approaches could be of value for yaws eradication efforts. In particular, this approach might allow a comparison of the differential effects of alternative mass treatment strategies, which would be difficult to assess by empirical randomized controlled trials because of the size and cost of implementing large-scale cluster randomized studies.

Previous mathematical models for yaws (14) have assessed the cost-effectiveness of yaws eradication but have not directly addressed the feasibility of achieving this goal or the number of rounds of treatment that would be 
required. In this study, we aimed to determine whether the eradication of yaws is feasible based on the Morges strategy and, if it is, the number and coverage of mass treatment rounds needed to achieve the goal.

\section{Methods}

We developed a stochastic Markov model of communitylevel transmission of yaws (Figure 1). This model treats each stage of the disease as a discrete compartment, with persons moving through each compartment as the disease progresses or is treated. Upon infection, susceptible persons acquire primary disease at a rate that is proportional to the transmission rate and the total number of infectious persons. Persons with primary disease can further transition to secondary disease, at which stage they remain infectious, and both those with primary and secondary disease can transition to latent disease, which is not infectious. Last, those with latent disease can relapse back to secondary infectious disease. The model includes a rate of routine treatment for persons with primary or secondary disease, after which they become susceptible to infection again. The model also includes a lower rate of routine treatment for latent disease, after which the patients also become susceptible to infection again. Unlike previous mathematical models of yaws (14), tertiary yaws was not included in the model because such cases are believed not to contribute to transmission (15). Because persons might be reinfected many times, we did not consider them to obtain protective immunity after infection or treatment (online Technical Appendix, http:// wwwnc.cdc.gov/EID/article/23/1/16-0487-Techapp1.pdf).

Although some evidence suggests the existence of a nonhuman primate reservoir for yaws in Africa (10), we did not include such infections in our model because there is currently no definitive evidence that the organism responsible for these infections is the same one that causes human yaws or that zoonotic transmission occurs in the real world. We therefore considered the epidemiologic importance of this possible reservoir as minimal when constructing our model.

\section{Population Size}

Estimates of the starting population for each compartment were derived from published population-based yaws prevalence studies $(7,17)$. We modeled a discrete closed population without addition or reduction through births or deaths.

\section{Disease Characteristic Variable Estimates}

We estimated values for the rates of disease progression between different stages of yaws, including development of and relapse from latent yaws, by using expert opinion, published data, and estimates used in other models (Table 1) $(1,14,17)$. We defined 3 transmission scenarios (low, medium, and high) by using published age-specific

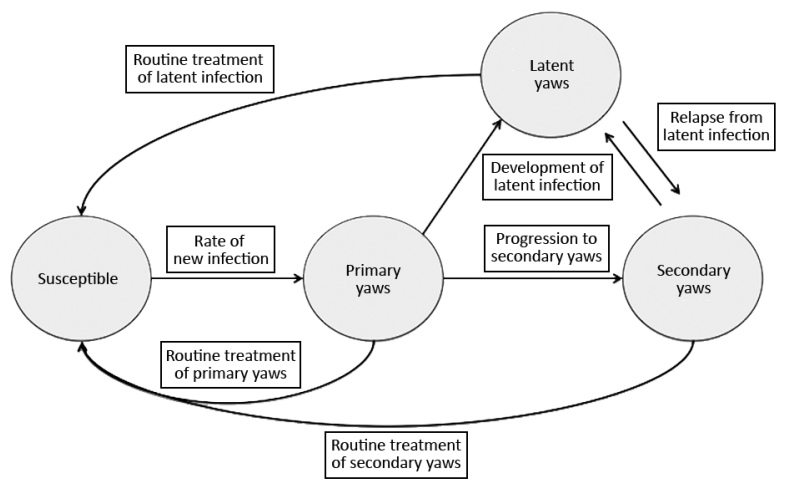

Figure 1. Markov model of yaws transmission. Susceptible persons become infected at a rate dependent on the probability of transmission and the number of persons with infectious primary and secondary yaws. Without treatment, illness progresses from primary disease to either latent yaws or secondary yaws. Persons with latent yaws might relapse to generate secondary cases of infectious yaws in others.

treponemal seroprevalence data (17), expert opinion, and values used in other yaws models (14). Based on these data, we calculated initial estimates of the number of new infections arising from a single index case in a fully susceptible population (the basic reproduction number $\left[\mathrm{R}_{0}\right]$ ). Based on the seroprevalence data, we generated $\mathrm{R}_{0}$ estimates of 1.25 (low), 1.83 (medium), and 2.4 (high). These estimates were converted to estimates of the probability of transmission after contact between an infectious person and a susceptible persons $(\beta)$. The mean $\mathrm{R}_{0}$ taking account of the full structure of our model, including duration of infection and the size of each starting population, is 1.96, resulting in a mean $\mathrm{R}_{0}$ of 1.45 (95\% CI 1.01-2.14) for the low transmission settings, 1.95 (95\% CI 1.38-2.91) for medium, and 2.47 (95\% CI 1.7-3.68) for high transmission. We included a variable to represent the likelihood of a person receiving treatment for yaws in the absence of public health interventions based on published data (17).

\section{Mass Drug Administration Variables Estimates}

We performed simulation experiments to estimate the impact of a yaws eradication intervention on disease transmission. In line with the Morges strategy (5), we considered 2 program components. In the first component, TCT, all persons were considered to have an equal chance of receiving treatment regardless of their infection status. In the second component, TTT, we considered that the coverage achieved among persons with active infection and those with latent infection might differ. Intervention coverage was modeled independently for TCT, with TTT pertaining to persons with active infection and persons with latent infection over a range of plausible estimates $(65 \%-95 \%$ population coverage). Mass treatment compliance was simulated as a random, nonsystematic process (i.e., each person had the same 
Table 1. Parameters used in modeling treatment coverage required to achieve yaws eradication

\begin{tabular}{|c|c|c|c|}
\hline Parameter & $\begin{array}{l}\text { Parameter } \\
\text { estimate }\end{array}$ & Source of estimate & Comments \\
\hline \multicolumn{4}{|l|}{ Epidemiologic parameters } \\
\hline $\mathrm{R}_{0}$ * & $1.08-3.32$ & \multirow{4}{*}{$\begin{array}{c}\text { Derived from } \\
\text { published survey } \\
\text { data (17) } \\
\text { Derived from expert } \\
\text { opinion and } \\
\text { previously published } \\
\text { models }(1,14)\end{array}$} & \multirow{4}{*}{$\begin{array}{l}\text { The average number of new cases } \\
\text { occurring from a single index case in a } \\
\text { fully susceptible population } \\
\text { All untreated persons with primary disease } \\
\text { either develop secondary or latent stage } \\
\text { disease, and this occurs over a period of } \\
2-6 \text { mo. Untreated persons with latent } \\
\text { cases might relapse for a period of } \geq 5 \text { y } \\
\text { and become actively infectious again. }\end{array}$} \\
\hline $\begin{array}{l}\text { Monthly probability of progression from } \\
\text { primary to secondary disease without } \\
\text { treatment }\end{array}$ & $2.78 \%-5.56 \%$ & & \\
\hline $\begin{array}{l}\text { Monthly probability of progression from } \\
\text { infectious to latent disease without treatment }\end{array}$ & $13.9 \%-27.8 \%$ & & \\
\hline $\begin{array}{l}\text { Monthly probability of relapse from latent } \\
\text { disease to infectious stage without treatment }\end{array}$ & $1 \%-3 \%$ & & \\
\hline \multicolumn{4}{|l|}{ Population parameters } \\
\hline Susceptible at baseline & $64 \%$ & \multirow{4}{*}{$\begin{array}{l}\text { Derived from } \\
\text { published survey } \\
\text { data (17) }\end{array}$} & \multirow{4}{*}{$\begin{array}{l}\text { Data derived from multiple pre-mass drug } \\
\text { administration surveys conducted in } \\
\text { communities where yaws is endemic }\end{array}$} \\
\hline Primary yaws at baseline & $1.5 \%$ & & \\
\hline Secondary yaws at baseline & $1.5 \%$ & & \\
\hline Latent yaws at baseline & $33 \%$ & & \\
\hline \multicolumn{4}{|l|}{ Mass treatment parameters } \\
\hline Total community treatment coverage $\dagger$ & $65 \%-95 \%$ & \multirow{5}{*}{$\begin{array}{l}\text { Expert opinion and } \\
\text { published data on } \\
\text { coverage achieved } \\
\text { in other mass } \\
\text { treatment campaigns } \\
\quad(18)\end{array}$} & \multirow{5}{*}{$\begin{array}{c}\text { Coverage estimates were chosen to reflect } \\
\text { the range achieved in real-world mass } \\
\text { drug administration programs for other } \\
\text { neglected tropical diseases }\end{array}$} \\
\hline $\begin{array}{l}\text { Total targeted treatment coverage of persons } \\
\text { with active cases } \ddagger\end{array}$ & $65 \%-95 \%$ & & \\
\hline $\begin{array}{l}\text { Total targeted treatment coverage of persons } \\
\text { with latent cases } \ddagger\end{array}$ & $65 \%-95 \%$ & & \\
\hline No. rounds of total community treatment $\dagger$ & $1-3$ & & \\
\hline No. rounds of total targeted treatmentł & $0-5$ & & \\
\hline
\end{tabular}

chance of receiving treatment, with the likelihood of any 1 person receiving treatment being independent).

We varied the number of treatment rounds of TCT (1-3 rounds) and TTT ( $0-5$ rounds). Where $\geq 1$ rounds of TTT were implemented, these rounds followed the final round of TCT. In line with the Morges strategy and real-world pilot implementations $(5,7)$, rounds of mass treatment were spaced at 6-month intervals. We also conducted an analysis with rounds of treatment spaced at 12-month intervals to assess whether annual treatment might also be effective.

We derived estimates of the efficacy of single-dose treatment with azithromycin from randomized controlled trials of azithromycin for the treatment of yaws (Table 1) (4). After successful treatment, yaws lesions become noninfectious within 24 hours (1); therefore, we considered treatment to be immediately efficacious at the time of mass drug administration, with persons reverting to a susceptible state after treatment.

\section{Implementing the Model}

The model was implemented in R software (19). We performed repeated simulations across a range of assumptions about the rate of transmission (equivalent to low, medium, and high estimates of $\mathrm{R}_{0}$ ) and assumptions about mass treatment, varying the coverage and number of mass treatment rounds undertaken (Table 1).

For each combination of disease and intervention parameters, we performed 1,000 simulation experiments.
Within each combination of transmission and treatment assumptions, we varied other disease-specific variables (e.g., rate of progression and relapse and treatment in the absence of intervention) across the range of parameter estimates. The model was run for an initial period of 50 months to achieve a steady state with yaws eradication interventions modeled to commence after this initial period. The model then ran for a further 100 months (online Technical Appendix).

\section{Assessing Outcomes}

For each run of the model, we recorded whether eradication was achieved. Eradication was defined as no cases of infectious or latent yaws at the end of the model run. The eradication probability was defined as the percentage of runs within each permutation of model characteristics where eradication was achieved. All analyses were performed by using $\mathrm{R}$ version 3.2.2.

\section{Results}

The model generated a total of 6,174 simulations of variable mass drug administration strategies. Because each strategy was implemented across a range $(n=3)$ of assumptions about the force of infection, a total of 18,522 simulations were created. The probability of achieving local interruption of transmission varied substantially across estimates of the force of infection and mass drug administration characteristics. 
At the lowest estimates of the force of infection $\left(\mathrm{R}_{0}=\right.$ 1.45 ) and with treatment rounds at 6-month intervals, the minimum treatment thresholds required to have a transmission interruption probability of $\geq 80 \%$ were a coverage of $>75 \%$ of all populations and $\geq 8$ rounds of treatment ( 3 rounds of TCT followed by 5 rounds of TTT). Increasing the coverage to $85 \%$ reduced the total number of rounds required to 5 (1-3 rounds of TCT followed by $2-4$ rounds of TTT) (Figure 2; Table 2). For comparison, when the gap between treatment rounds was extended from 6 to 12 months, a total of 7 rounds of $85 \%$ coverage were required (2-3 rounds of TCT and 4-5 rounds of TTT).

At medium estimates of the force of infection $\left(\mathrm{R}_{0}=1.95\right)$ and with treatment rounds at 6-month intervals, the equivalent thresholds were $90 \%$ coverage and a total of 7 rounds of treatment (2-3 rounds of TCT and 4-5 rounds of TTT) (Figure 2; Table 2). When the gap between treatment rounds was increased to 12 months, no combination of treatment variables was predicted to have a transmission interruption probability of $\geq 80 \%$.

At the highest estimates of the force of infection $\left(\mathrm{R}_{0}=3.32\right)$ and with treatment rounds at 6-month intervals, a total of 8 rounds ( 3 rounds of TCT and 5 rounds of TTT) with $95 \%$ coverage were required for $\mathrm{a} \geq 80 \%$ likelihood of interrupting transmission (Figure 2; Table 2). When the gap between treatment rounds was increased to 12 months, no combination of treatment variables was predicted to have a probability of interrupting transmission of $\geq 80 \%$.

We considered it plausible that, under field conditions, the coverage of persons with latent infection would not exceed $70 \%$ in any given round of TTT, because such cases are not clinically apparent, and adequate coverage might not be achieved by treating the immediate contacts of persons with clinical infection. At lower estimates of the force of infection, a total of 3 rounds of TCT with $85 \%$ coverage and 3 rounds of TTT (each with a coverage of persons with active infection of $85 \%$ and coverage of persons with latent infection of $65 \%$ ) was associated with $a \geq 80 \%$ probability of interrupting transmission. If only 1 round of TCT was conducted, then coverage during TCT needed to be $90 \%$ and a total of 5 rounds of TTT (each with $90 \%$ coverage of persons with active infection and $65 \%$ coverage of persons with latent infection) were required. For medium estimates of the force of infection, a total of 8 rounds of treatment ( 3 rounds of TCT and 5 rounds of TTT) with a coverage of $90 \%$ were required. If only 1 round of TCT was undertaken, then $95 \%$ coverage was required, followed by 5 rounds of TTT with a $95 \%$ coverage of persons with active infection and $70 \%$ coverage of persons with latent infection. Under the highest estimate of the force of infection, no combination of treatment variables was associated with a high probability of interrupting transmission.

\section{Discussion}

Our study demonstrates that, with implementation of the Morges strategy, interruption of transmission is possible in the setting considered. This finding suggests that eradication of yaws could be achieved, although caution must be applied because variability in the parameter estimates elsewhere could affect the effectiveness of these strategies. The parameter that has the strongest influence on whether elimination can be achieved is the transmission rate; that is, the rate at which infection occurs given contact between a

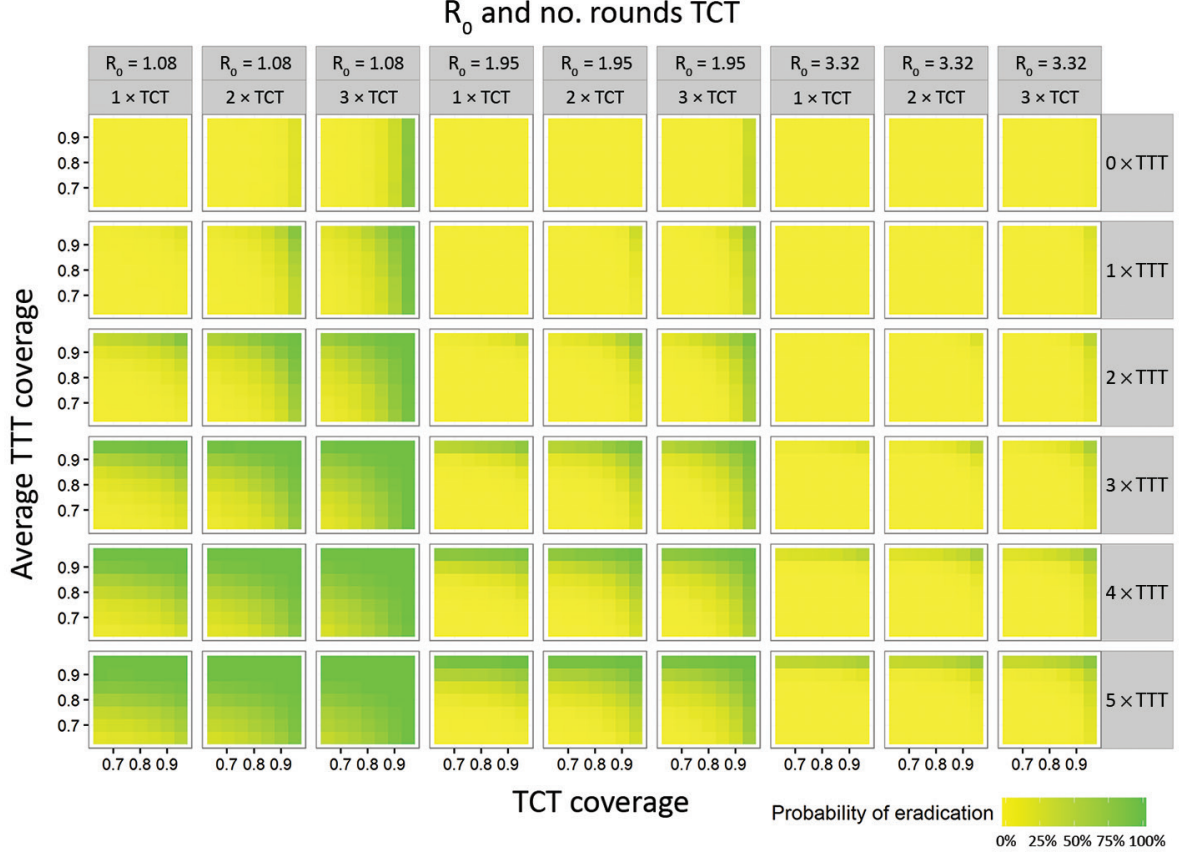

Figure 2. Predicted probability of achieving yaws eradication given variations in the estimate of $\mathrm{R}_{0}$ (basic reproduction number), total community treatment coverage, number of rounds of total community treatment, total targeted treatment coverage (TTT), and number of rounds of

$z$ TTT. For this graph, we only show simulations where the coverage $\vec{O}$ of persons with latent cases is the 5 same as the coverage of persons with active cases during TTT. This might overrepresent the actual likelihood of achieving eradication because the coverage of persons with latent cases is probably lower than the coverage of persons with active cases during TTT. $R_{0}$, basic reproduction number; TCT, total community treatment; TTT, total targeted treatment. 
Table 2. Indicative predicted coverage and number of rounds of treatment required to achieve yaws eradication

\begin{tabular}{|c|c|c|c|c|}
\hline \multirow[b]{2}{*}{ Predicted probability by estimated $\mathrm{R}_{0}$} & \multicolumn{2}{|c|}{ Treatment every $6 \mathrm{mo}$} & \multicolumn{2}{|c|}{ Treatment every $12 \mathrm{mo}$} \\
\hline & Coverage required & Total no. rounds* & Coverage required & Total no. rounds* \\
\hline \multicolumn{5}{|l|}{$80 \%$ predicted probability of eradication } \\
\hline Low $\mathrm{R}_{0}(1.45,95 \%$ Cl 1.01-2.14) & $75 \%$ & 8 & $85 \%$ & 7 \\
\hline Medium $\mathrm{R}_{0}(1.95,95 \% \mathrm{Cl} 1.38-2.91)$ & $90 \%$ & 7 & $\dagger$ & $\dagger$ \\
\hline High $\mathrm{R}_{0}(2.47,95 \% \mathrm{Cl} 1.7-3.68)$ & $95 \%$ & 8 & $\dagger$ & $\dagger$ \\
\hline \multicolumn{5}{|l|}{$100 \%$ predicted probability of eradication } \\
\hline Low $\mathrm{R}_{0}(1.45,95 \% \mathrm{Cl} 1.01-2.14)$ & $85 \%$ & 8 & $95 \%$ & 6 \\
\hline Medium $\mathrm{R}_{0}(1.95,95 \% \mathrm{Cl} 1.38-2.91)$ & $\dagger$ & $\dagger$ & $\dagger$ & $\dagger$ \\
\hline High $\mathrm{R}_{0}(2.47,95 \% \mathrm{Cl} 1.7-3.68)$ & $\dagger$ & $\dagger$ & $\dagger$ & $\dagger$ \\
\hline \multicolumn{5}{|c|}{$\begin{array}{l}{ }^{*} \mathrm{R}_{0} \text { (basic reproduction number) is the number of new cases arising from a single index case in a fully susceptible population. For this table, the number } \\
\text { of rounds of total community treatment and total targeted treatment is combined (e.g., a total of } 7 \text { rounds could be } 3 \text { rounds of total community treatment } \\
\text { and } 4 \text { rounds of total targeted treatment or, alternatively, } 2 \text { rounds of total community treatment and } 5 \text { rounds of total targeted treatment). Data are } \\
\text { indicative only, and in some settings, higher coverage would allow a reduction in the total number of rounds required (see text and Figure 2). } \\
\text { †No combination of treatment variables was associated with the stated eradication probability. }\end{array}$} \\
\hline
\end{tabular}

susceptible and an infectious person (online Technical Appendix Figure). We considered 3 different scenarios of the transmission rate of yaws based on serologic data, and our estimate of the feasibility of elimination varied considerably depending on these estimates. Further studies to obtain better estimates of the $\mathrm{R}_{0}$ in a range of countries where yaws is endemic would be of value to inform improved models and programmatic planning.

A minimum of 8 rounds with coverage of $\geq 75 \%$ seems to be required for a high likelihood of achieving eradication but would prove inadequate at our highest estimates of possible values for $\mathrm{R}_{0}$. The predictions of our model are broadly in keeping with the real-world findings of the successful yaws elimination program in India (9), where 7 years of consecutive case finding and treatment (analogous to 14 rounds of TTT with $75 \%$ coverage) were conducted. In our model, the number of rounds of TTT also had a marked effect on the likelihood of achieving eradication, especially when coverage of persons with latent cases was limited to $\leq 70 \%$. In these settings, the required number of rounds of treatment to interrupt transmission increased considerably.

Relatively few data are available on the transmission rate of yaws. Even within yaws-endemic countries, the prevalence of yaws varies markedly. Studies in the Pacific have found a seroprevalence of antitreponemal antibodies of $>30 \%$ in several communities $(7,17)$ and a prevalence of clinical yaws of ranging from $2.5 \%$ to $5 \%$ in communities before mass treatment. The prevalence of yaws is markedly lower in many yaws-endemic countries in West Africa (20), but limited community-based seroprevalence data are available to inform our understanding of disease transmission there.

We modeled a range of estimates of $\mathrm{R}_{0}$ from 1.08 to 3.32 based on seroprevalence data and expert opinion. Given the substantial influence of these estimates on the likely outcome of community mass treatment, further studies to better understand disease transmission and how this varies within and between endemic communities would be of value. Ideally, these studies would obtain communitylevel, age-specific seroprevalence data that could be used to calculate the force of infection. No perfect serologic marker can be used for this task. Traditional treponemal serology combines a treponemal test, which reflects lifetime exposure but remains positive for life, with a nontreponemal test, the titer of which rises and falls after treatment. It is therefore not possible to use seroprevalence data to distinguish persons who have been infected many times from those who have been infected once, and seroprevalence estimates are likely to underestimate the actual force of infection. For this study, we calculated the force of infection while relying on treponemal serology alone, which should provide a more accurate estimate of the force of infection than if we used dual-positive serology. It remains, however, an imperfect measure.

Our model predicts that high coverage is required in all rounds of treatment to make yaws eradication feasible. Data from the previous WHO and United Nations Children's Fund mass treatment campaign have highlighted the importance of achieving high coverage of persons with latent cases of yaws (21) and that treatment of persons with active cases alone is insufficient to interrupt transmission. These factors were important considerations in the adoption as part of the Morges strategy of an initial round of TCT regardless of the prevalence of active disease in a community. Given the high coverage requirement, particularly of persons with latent cases, and the relatively high fixed-costs of reaching yaws-endemic communities (14) compared with the relatively low costs of generic azithromycin, it might be preferable to conduct multiple rounds of TCT before the switch to TTT. Such a recommendation would be in line with the original Morges strategy (22), which recommended that additional rounds of TCT could be considered if the coverage achieved in the initial round of treatment was $<90 \%$ or if access to yaws-endemic communities was difficult. A switch to multiple rounds of community mass treatment might also facilitate integration with other neglected tropical diseases mass drug administration programs in countries that are also frequently based on whole community mass treatment (23), although our model predicted a higher probability of achieving eradication with biannual 
treatment. Further studies to help determine the optimum strategy for achieving high coverage of persons with latent cases during the TTT phase of eradication efforts should be considered (e.g., studies of the spatial epidemiology of latent yaws cases in relation to persons with active cases in both pre- and post-mass drug administration settings or studies of whether additional mass treatment rounds specifically targeting children might be beneficial).

Our study has several limitations. Most notably, we lack accurate estimates for several disease parameters. The parameters used are derived from expert opinion and data from the Pacific region, and the transmission dynamics of yaws might be different in other regions of the world. However, the disease parameters used in this study are broadly in line with those used by other models of yaws transmission (14). We tested a range of coverage estimates for community mass treatment, but we did not factor in the possibility that some persons might be systematically missed during mass treatment campaigns, a phenomenon that has been observed in control efforts for other neglected tropical diseases (24). The current Morges strategy does not include adjunctive elements, such as water, sanitation, and hygiene interventions, in addition to mass treatment, although some studies suggest that improved access to water and sanitation is associated with a decreased risk for yaws (17). We did not include a secular trend in our model, and such a trend could be anticipated to further increase the likelihood of yaws eradication being achieved. Our model was designed to assess the feasibility of achieving yaws eradication in the near future, driven by the current WHO strategy, and in those conditions any effect of a secular trend could be expected to be minimal compared with the substantial impact of community mass treatment. Previous models have shown that secular trends are unlikely to substantially affect the costeffectiveness of mass treatment (14); however, those models were based on an assumption of $90 \%-99 \%$ coverage in a single TCT round and $100 \%$ coverage of index patients and their contacts in the TTT round. More generally, we used a single-model structure that is simplified by modeling persons as being in 1 of a small number of disease-related compartments at any time and considering contact to occur at random. Uncertainty in model structure relating to disease progression and the probability of contact means that our findings should be interpreted carefully and potentially reassessed as elimination strategies are being applied.

In conclusion, our study assessed the theoretical achievability of worldwide yaws eradication and represents an important milestone in reaching the WHO's eradication target. We have defined programmatic thresholds that might need to be met to achieve yaws eradication and identified key research questions to be addressed to inform refinements of the model and the worldwide roll-out of treatment strategies.
M.M. is supported by the Wellcome Trust (grant no. 102807).

Dr. Marks is a Wellcome Trust Clinical Research Fellow at the London School of Hygiene \& Tropical Medicine. His primary research interests include strategies to eradicate yaws and the development of integrated approaches to the control and elimination of neglected tropical diseases.

\section{References}

1. Mitjà $\mathrm{O}$, Asiedu K, Mabey D. Yaws. Lancet. 2013;381:763-73. http://dx.doi.org/10.1016/S0140-6736(12)62130-8

2. Kazadi WM, Asiedu KB, Agana N, Mitjà O. Epidemiology of yaws: an update. Clin Epidemiol. 2014;6:119-28. http://dx.doi.org/10.2147/CLEP.S44553

3. Asiedu K, Fitzpatrick C, Jannin J. Eradication of yaws: historical efforts and achieving WHO's 2020 target. PLoS Negl Trop Dis. 2014;8:e3016. http://dx.doi.org/10.1371/journal.pntd.0003016

4. Mitjà O, Hays R, Ipai A, Penias M, Paru R, Fagaho D, et al. Single-dose azithromycin versus benzathine benzylpenicillin for treatment of yaws in children in Papua New Guinea: an open-label, non-inferiority, randomised trial. Lancet. 2012;379:342-7. http://dx.doi.org/10.1016/S0140-6736(11)61624-3

5. World Health Organization. Eradication of yaws - the Morges strategy. Wkly Epidemiol Rec. 2012;87:189-94.

6. World Health Organization. World Health Assembly resolution 66.12 [cited 2016 Mar 21]. http://www.who.int/neglected_diseases/ mediacentre/WHA_66.12_Eng.pdf

7. Mitjà $\mathrm{O}$, Houinei $\overline{\mathrm{W}}$, Moses $\mathrm{P}$, Kapa A, Paru R, Hays R, et al. Mass treatment with single-dose azithromycin for yaws. N Engl J Med. 2015;372:703-10. http://dx.doi.org/10.1056/NEJMoa1408586

8. Marks M, Vahi V, Sokana O, Chi KH, Puiahi E, Kilua G, et al. Impact of community mass treatment with azithromycin for trachoma elimination on the prevalence of yaws. PLoS Negl Trop Dis. 2015;9:e0003988. http://dx.doi.org/10.1371/journal.pntd.0003988

9. World Health Organization. Elimination of yaws in India. Wkly Epidemiol Rec. 2008;83:125-32.

10. Marks M, Mitjà O, Vestergaard LS, Pillay A, Knauf S, Chen CY, et al. Challenges and key research questions for yaws eradication. Lancet Infect Dis. 2015;15:1220-5. http://dx.doi.org/10.1016/ S1473-3099(15)00136-X

11. Anderson R, Truscott J, Hollingsworth TD. The coverage and frequency of mass drug administration required to eliminate persistent transmission of soil-transmitted helminths. Philos Trans R Soc Lond B Biol Sci. 2014;369:20130435. http://dx.doi.org/10.1098/rstb.2013.0435

12. Anderson RM, Turner HC, Truscott JE, Hollingsworth TD, Brooker SJ. Should the goal for the treatment of soil transmitted helminth (STH) infections be changed from morbidity control in children to community-wide transmission elimination? PLoS Negl Trop Dis. 2015;9:e0003897. http://dx.doi.org/10.1371/journal. pntd.0003897

13. Stolk WA, ten Bosch QA, de Vlas SJ, Fischer PU, Weil GJ, Goldman AS. Modeling the impact and costs of semiannual mass drug administration for accelerated elimination of lymphatic filariasis. PLoS Negl Trop Dis. 2013;7:e1984. http://dx.doi.org/ 10.1371/journal.pntd.0001984

14. Fitzpatrick C, Asiedu K, Jannin J. Where the road ends, yaws begins? The cost-effectiveness of eradication versus more roads. PLoS Negl Trop Dis. 2014;8:e3165. http://dx.doi.org/10.1371/ journal.pntd.0003165

15. Marks M, Mitjà O, Solomon AW, Asiedu KB, Mabey DC. Yaws. Br Med Bull. 2015;113:91-100. http://dx.doi.org/10.1093/bmb/ldu037

16. Knauf S, Liu H, Harper KN. Treponemal infection in nonhuman primates as possible reservoir for human yaws. Emerg Infect Dis. 2013;19:2058-60. http://dx.doi.org/10.3201/eid1912.130863 
17. Marks M, Vahi V, Sokana O, Puiahi E, Pavluck A, Zhang Z, et al. Mapping the epidemiology of yaws in the Solomon Islands: a cluster randomized survey. Am J Trop Med Hyg. 2015;92:129-33. http://dx.doi.org/10.4269/ajtmh.14-0438

18. Worrell C, Mathieu E. Drug coverage surveys for neglected tropical diseases: 10 years of field experience. Am J Trop Med Hyg. 2012;87:216-22. http://dx.doi.org/10.4269/ajtmh.2012.12-0167

19. Johnson P. Adaptivetau: Tau-leaping stochastic simulation. R package version 2.2 [cited 2016 Mar 21]. https://CRAN.Rproject.org/package $=$ adaptivetau

20. Mitjà $\mathrm{O}$, Marks M, Konan DJP, Ayelo G, Gonzalez-Beiras C, Boua B, et al. Global epidemiology of yaws: a systematic review. Lancet Glob Health. 2015;3:e324-31. http://dx.doi.org/10.1016/ S2214-109X(15)00011-X

21. Hackett CJ, Guthe T. Some important aspects of yaws eradication. Bull World Health Organ. 1956;15:869-96.
22. World Health Organization. Summary report of a consultation on the eradication of yaws, 5-7 March 2012, Morges, Switzerland [cited 2016 Jan 4]. http://www.who.int/iris/handle/10665/75528

23. Solomon AW, Marks M, Martin DL, Mikhailov A, Flueckiger RM, Mitjà $\mathrm{O}$, et al. Trachoma and yaws: common ground? PLoS Negl Trop Dis. 2015;9:e0004071. http://dx.doi.org/10.1371/journal. pntd.0004071

24. Edwards T, Allen E, Harding-Esch EM, Hart J, Burr SE, Holland MJ, et al. Non-participation during azithromycin mass treatment for trachoma in the Gambia: heterogeneity and risk factors. PLoS Negl Trop Dis. 2014;8:e3098. http://dx.doi.org/10.1371/journal.pntd.0003098

Address for correspondence: Michael Marks, Clinical Research Department, London School of Hygiene and Tropical Medicine, Keppel St, London, WC1E 7HT, UK; email: michael.marks@1shtm.ac.uk

\title{
etymologia
}

\section{Bayesian Probability}

\author{
Ronnie Henry, Martin I. Meltzer
}

$T^{1}$ homas Bayes (1701-1761) was a Presbyterian Minister, and how he become interested in statistics and probability is uncertain. Bayes presented his famous theorem on probability in "An Essay Towards Solving a Problem in the Doctrine of Chances," which was published posthumously by his friend Richard Price in 1763. Bayes's theorem provides a method of explicitly including prior events or knowledge when considering the probabilities of current events (for example, including a history of smoking when calculating the probability of developing lung cancer). Bayesian approaches use prior knowledge and information (e.g., probabilities) that may help reduce uncertainty in analysis and have therefore been increasingly adopted by analysts in public health.

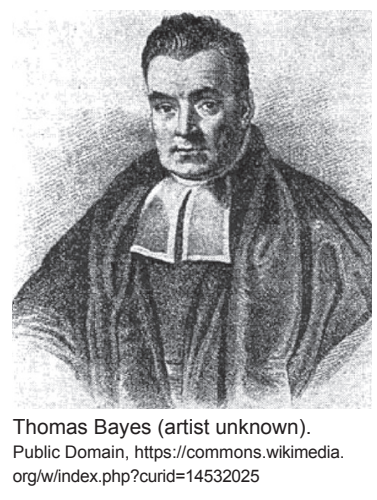

\section{Sources}

1. Armitage P, Berry G. Statistical methods in medical research. London: Blackwell Scientific Publications; 1994.

2. McGrayne SB. The theory that would not die: how Bayes' rule cracked the enigma code, hunted down
Russian submarines and emerged triumphant from two centuries of controversy. New Haven (CT): Yale University Press; 2011.

3. Barnard GA. Thomas Bayes - a biographical note. Biometrika. 1958;45:293-5. http://dx.doi.org/10.1093/ biomet/45.3-4.293 Address for correspondence: Ronnie Henry, Centers for Disease Control and Prevention, 1600 Clifton Rd NE, Mailstop E03,
Atlanta, GA 30329-4027, USA; email: boq3@cdc.gov

DOI: http://dx.doi.org/10.3201/eid2301.ET2301 